Neutrino Physics--Theoretical Considerations *

\author{
BENJAMIN W. $\mathrm{LEE}^{\dagger}$ \\ National Accelerator Laboratory, Batavia, Illinois 60510
}

\footnotetext{
* Talk presented at the IVth International Conference on Neutrino Physics and Astrophysics, Philadelphia, Pennsylvania, April 26-28, 1974.

${ }^{\dagger}$ On leave of absence from the Institute for Theoretical Physics, State University of New York, Stony Brook, NY 11790.
} 


\section{NEUTRINO PHYSICS-THEORETICAI, CONSIDERATIONS}

Benjamin W. Lee

National Accelerator Laboratory, Batavia, IL. 60510

\section{O. PROLOGUE}

Neutrino physics is of interest not only for the study of intrinsic characteristics of the elusive neutrinos, but also as diagnostics of hadronic structures, and as a probe of weak interactions.

What follows is a more or less faithful reproduction of the transparencies used in the oral presentation. In preparing this talk, I relied heavily on the following excellent reviews:

S. Adler, "Accelerator Neutrino Physics, Present and Future, "NAL-Conf-74/39-THY,

C. H. Llewellyn-Smith, Physics Reports 3C, No. 5 (1972),

E.A. Paschos, NAL-Conf-73/65-THY: Lectures delivered at the "Ettore Majorana" Summer School, 1973.

For comprehensive bibliographies, the above $r$ eferences should be consulted.

I have used a number of figures and illustrations from published papers available before this Conference. More up-to-date data have been presented at the Conference and the reader should consult other contributions in these Proceedings for these.

\section{KINEMATICS AND STANDARD V-A THEORY}

1. Kinematics.

$$
v\left(\mathrm{k}_{1}\right)+\mathrm{A}(\mathrm{p}) \rightarrow \ell\left(\mathrm{k}_{2}\right)+\mathrm{B}(\mathrm{p}+\mathrm{q})
$$

where $q=k_{1}-k_{2}$ is the momentum transfer.

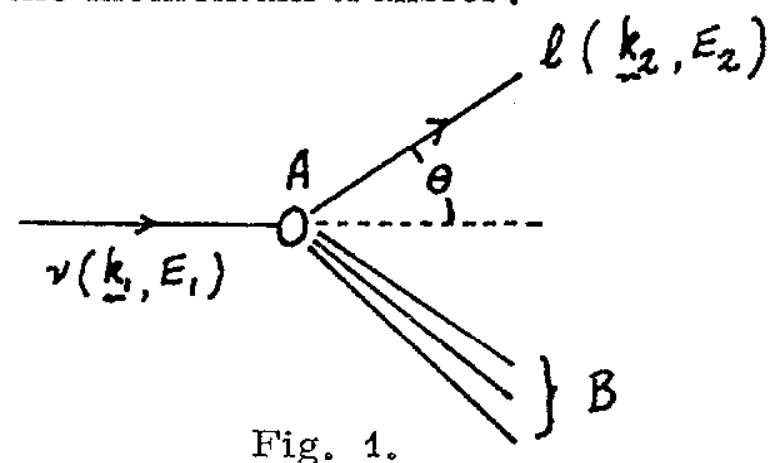

Kinematics of neutrino-induced reactions.

Invariant variables:

$$
v=p \cdot q=m_{A}\left(E_{1}-E_{2}\right)
$$




$$
\mathrm{q}^{2}=\left(\mathrm{k}_{1}-\mathrm{k}_{2}\right)^{2}=-4 \mathrm{E}_{1} \mathrm{E}_{2} \sin ^{2} \frac{\theta}{2} \text { (neglecting } \ell \text { mass). }
$$

2. V-A Theory.

Local Current $\times$ Current Interaction:

$$
\begin{aligned}
& \mathscr{L}_{\mathrm{eff}}=\frac{\mathrm{G}_{\mathrm{F}}}{\sqrt{2}} \mathscr{f}_{\mu}^{\dagger} \mathscr{F}^{\mu}, \mathrm{G}_{\mathrm{F}} \simeq 10^{-5} \mathrm{~m}_{\mathrm{p}}^{-2} . \\
& \mathscr{F}_{\mu}=J_{\mu}+\ell \ell_{\mu} \\
& J_{\mu}=\left(V_{\mu}^{1+i 2}-A_{\mu}^{1+i 2}\right) \cos \theta_{c}+\left(V_{\mu}^{4+i 5}-A_{\mu}^{4+i 5}\right) \sin \theta_{c}, \\
& \ell_{\mu}=\bar{\nu} \gamma_{\mu}\left(1-\gamma_{5}\right) e+\bar{\nu} \gamma_{\mu}\left(1-\gamma_{5}\right) \mu, \\
& \theta_{\mathrm{c}} \cong 15^{\circ}=\pi / 12 \text {. }
\end{aligned}
$$

and

\section{Current Algebra:}

Normalization of $\mathrm{V}_{\mu}^{\mathrm{i}}$ and $\mathrm{A}_{\mu}^{i}$ is fixed by Gell-Mann's SU(3) $\times$
algebra: SU(3) algebra:

$$
\left[A_{0}^{i}(\vec{x}, 0), A_{0}^{j}(\vec{y}, 0)\right]=i f^{i j k} V_{0}^{k}(\vec{x}, 0) \delta^{3}(\underset{\sim}{x}-\underset{\sim}{y}),
$$

etc.

$$
\begin{aligned}
& \text { G-parity: } \quad G=C e^{i \pi I_{2}} . \\
& G\left\{\begin{array}{l}
V^{1+i 2} \\
A^{1+i 2}
\end{array}\right\} G^{-1}=\left\{\begin{array}{l}
+V^{1+i 2} \\
-A^{1+i 2}
\end{array}\right\} \text { i. e., 1st class. }
\end{aligned}
$$

Existence of the 2nd class currents not ruled out . this conference.

Lepton Number Conservation: See S. P. Rosen's discussion at

\section{EXCLUSIVE REACTIONS}

1. Quasi-elastic: $A=N, B=N$.

$$
\nu_{\mu}+n \rightarrow \mu^{-}+p
$$


Hadronic matrix element:

$$
\left.<p\left(p_{2}\right)\left|J_{\mu}\right| n\left(p_{1}\right)\right\rangle=\cos \theta_{c} \bar{u}_{p}\left(p_{2}\right) \Gamma_{\mu} u_{n}\left(p_{1}\right)
$$

where

$$
\begin{aligned}
& \Gamma_{\mu}=\gamma_{\mu} F_{V}^{1}\left(q^{2}\right)+\frac{i \sigma_{\mu \nu} q^{\nu}}{2 m_{N}} F_{V}^{2}\left(q^{2}\right)+\frac{q_{\mu}}{2 m_{N}} F_{V}^{3}\left(q^{2}\right) \\
& -\gamma_{\mu} \gamma_{5} G_{A}\left(q^{2}\right)-\gamma_{5} q_{\mu} H_{A}\left(q^{2}\right)+\frac{1}{m_{N}} \gamma_{5}\left(p_{1}+p_{2}\right)_{\mu} F_{A}^{3}\left(q^{2}\right) .
\end{aligned}
$$

Absence of 2nd class current implies $F_{V, A}^{3}=0$; CVC implies $F_{V}^{3}=0$, and $F_{V}^{1,2}$ are as in ep scattering. Since $q^{\lambda} \bar{\mu} \gamma_{\lambda}\left(1-\gamma_{5}\right) \nu \propto m_{\mu}{ }^{\prime} H_{A}$ may be neglected. Parameterize $\mathrm{GA}_{\mathrm{A}}=1.24\left(1-\mathrm{q}^{2} / \mathrm{M}^{\mathrm{A}} \mathrm{A}^{2}\right)^{-2}$.

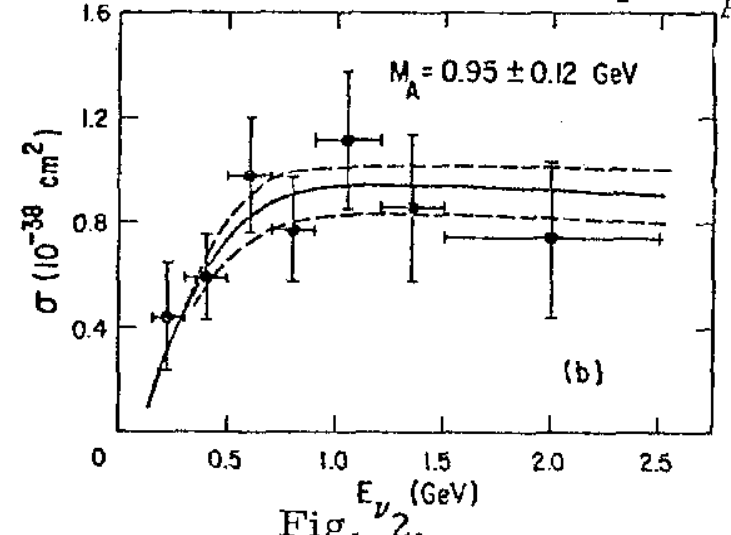

Cross Section for $\nu_{\mu}+\mathrm{n} \rightarrow \mu^{-}+\mathrm{p}$ and fit with $\mathrm{M}_{\mathrm{A}}=0.95 \pm$ $0.12 \mathrm{GeV}$. From W.A. Mann, et al., Phys. Rev. Letters 31, 844 (1973). See also P. Schreiner's contribution.

2. $\triangle(1236)$ Production: $A=N, B=\Delta\left(1236, P_{3 / 2}, 3 / 2\right)$.<smiles>[13CH2]N[15NH2]</smiles>

Adler's theory [Ann. Phys., 50, 189 (1968)] : Relativistic version of the static model. Non-resonant amplitudes $=$
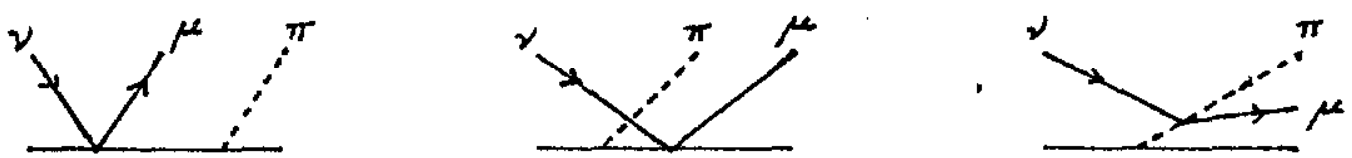

Fig. 3.

Born diagrams in Adler's theory. 
Born approximations; Resonant multipoles = Born approximation + rescattering corrections via $\pi \mathrm{N}$ phase shifts. Complete and

essentially unique predictions in terms of $\delta_{\ell \pm}, F_{V}^{1,2}, G_{A}$ and $H_{A}$. Works o.k. for photoproduction (CGLN); for electroproduction $\left(\mathrm{q}^{2} \leqslant 1 \mathrm{GeV}\right)$ and also for $v$ production. For comparison with experiment, see J. Campbell et al., Phys. Rev. Letters 8, 335 (1973); P. Schreiner et al. , ibid., 8, 339 (1973); P. Schreiner, in these Proceedings.

\section{INCLUSIVE REACTIONS}

\section{Kinematics.} section is

$A=N, B=$ sum over all final states for fixed $m_{B^{\circ}}$ The cross

$$
\sigma^{v, \bar{v}}=\mathrm{L}^{\alpha \beta}(\nu, \bar{v}) \mathrm{H}_{\alpha \beta}^{\nu, \bar{v}}
$$

where

$$
\begin{aligned}
& \mathrm{H}_{\alpha \beta}^{v}=\frac{1}{2} \sum_{B} \sum_{\text {spin }}\left\langle\mathrm{N}\left|\mathrm{J}^{\dagger}\right| \mathrm{B}\right\rangle\left\langle\mathrm{B}\left|\mathrm{J}_{\beta}\right| \mathrm{N}\right\rangle \\
& \text { A } \\
& \times(2 \pi)^{3} \delta^{4}\left(q+p-p_{B}\right) \\
& \left(v \rightarrow \bar{v}, J \leftrightarrow J^{\dagger}\right) \\
& =-q_{\alpha \beta} W_{1}+\frac{p_{\alpha} p_{\beta}}{m_{N}^{2}} W_{2}-i \frac{1}{2 m_{N}^{2}} \epsilon_{\alpha \beta \rho \sigma} p^{p_{q}{ }^{\sigma}} W_{3} \\
& +\frac{\mathrm{q}_{\alpha} \mathrm{q}_{\beta}}{\mathrm{m}_{\mathrm{N}}^{2}} \mathrm{w}_{4}+\frac{1}{2 \mathrm{~m}_{\mathrm{N}}^{2}}\left(\mathrm{p}_{\alpha} \mathrm{q}_{\beta}+\mathrm{p}_{\beta \mathrm{q}_{\alpha}}\right) \mathrm{w}_{5} \\
& +i \frac{1}{2 m_{N}^{2}}\left(p_{\alpha} q_{\beta}-p_{\beta} q_{\alpha}\right) w_{6} \text {. }
\end{aligned}
$$




$$
\begin{gathered}
\frac{d^{2} \sigma}{d\left|q^{2}\right| d v}=\frac{G_{F}^{2}}{2 \pi m_{N}^{2}}\left(\frac{E_{\mu}}{E_{\nu}}\right)\left[\cos ^{2} \frac{\theta}{2} W_{2}+2 \sin ^{2} \frac{\theta}{2} W_{1}\right. \\
\left.\mp \frac{E_{\mu}+E_{\nu}}{m_{N}} \sin ^{2} \frac{\theta}{2} W_{3}+O\left(m_{\mu}^{2}\right)\right] \text { for } \frac{v}{v} .
\end{gathered}
$$

Charge symmetry constraints $\left(\theta_{c}=0\right)$ :

$$
\begin{gathered}
e^{i \pi I} J_{\mu} e^{-i \pi I} 2=-J_{\mu}^{\dagger} \Rightarrow W_{i}^{\nu n}=W_{i}^{\overline{\nu p}}, \\
w_{i}^{\nu p}=w_{i}^{\bar{\nu} n} .
\end{gathered}
$$

2. Current algebra prediction.

$$
\begin{aligned}
& {\left[\int d^{3} x J^{0}(\vec{x}, 0) e^{i q \cdot x}, \int d^{3} y J^{0 \dagger}(\vec{y}, 0)\right] } \\
= & 4 I_{3} \cos ^{2} \theta_{c}+\left(3 y+2 I_{3}\right) \sin ^{2} \theta_{c}+\ldots
\end{aligned}
$$

Take spin averaged matrix element between nucleons and go to infinite momentum frame $\Rightarrow$

Adler sum rule:

$$
\begin{aligned}
& \frac{1}{\mathrm{~m}_{\mathrm{N}}^{2}} \int_{0}^{\infty} \mathrm{d} v\left[\mathrm{~W}_{2}^{\bar{v}}\left(v, \mathrm{q}^{2}\right)-\mathrm{W}_{2}^{\nu}\left(v, \mathrm{q}^{2}\right)\right] \\
= & <4 \cos ^{2} \theta_{\mathrm{c}} \mathrm{I}_{3}+\left(3 \mathrm{y}+2 \mathrm{I}_{3}\right) \sin ^{2} \theta_{\mathrm{c}}>\mathrm{N} \\
\simeq & 2 \text { for } \mathrm{N}=\mathrm{p} \mid \text { independent of } \mathrm{q}^{2 ! !}
\end{aligned}
$$

Alternatively 


$$
\begin{array}{cc}
-7- & \text { NAL-Conf-74/50-THY } \\
\lim _{\nu \rightarrow \infty}\left[\frac{d \sigma^{\nu p}}{d\left|q^{2}\right|}-\frac{d \sigma^{\nu p}}{d\left|q^{2}\right|}\right]=-\frac{G_{F}}{\pi}\left(\cos ^{2} \theta_{c}+2 \sin ^{2} \theta_{c}\right)
\end{array}
$$

3. Scaling

Dimensional analysis gives

$$
\begin{gathered}
W_{1}\left(v, q^{2}\right)=G_{1}\left(x,\left.|q|\right|^{2} / m_{N}^{2}\right), \\
\frac{v}{m_{N}^{2}} W_{2}\left(v, q^{2}\right)=G_{2}\left(x,\left|q^{2}\right| / m_{N}^{2}\right), \\
\frac{v}{m_{N}^{2}} W_{3}\left(v, q^{2}\right)=G_{3}\left(x,\left|q^{2}\right| / m_{N}^{2}\right) .
\end{gathered}
$$

Convenient scaling variables are

$$
\begin{gathered}
x=-g^{2} / 2 v \equiv \omega^{-1}, y=\frac{v}{m_{N} E_{\nu}}=\left(1-\frac{E_{\mu}}{E_{\nu}}\right) \cong \frac{E_{B}}{E_{\nu}}, \\
0 \leq x, y \leq 1 .
\end{gathered}
$$

[ Note: $z=x y=|q|^{2} / 2 m_{N} E_{\nu}=2\left(\frac{E}{m_{N}}\right) \sin ^{2} \frac{\theta}{2}$ is scale-invariant.]

$$
\begin{aligned}
\frac{d^{2} \sigma}{d x d y} & =\frac{G^{2} m_{N} E_{\nu}}{\pi}\left[\left(1-y-\frac{1}{2} x y \frac{m_{N}}{E_{\nu}}\right) G_{2}\right. \\
& \left.+x y^{2} G_{1} \mp x y\left(1-\frac{1}{2} y\right) G_{3}\right] .
\end{aligned}
$$

Bjorken scaling hypothesis:

$$
G_{i}\left(x,\left|q^{2}\right| / m_{N}^{2}\right) \underset{\substack{\nu, q^{2} \rightarrow \infty \\ x \text { fixed }}}{\longrightarrow} F_{i}(x) .
$$

In Bj limit, 


$$
\left(\frac{\pi}{G_{F}^{2} m_{N} E_{V}}\right) \frac{d^{2} \sigma}{d x d y}=x y^{2} F_{1}(x)+(1-y) F_{2} \mp x y\left(1-\frac{1}{2} y\right) F_{3} .
$$

Positivity considerations, $\epsilon^{\alpha} \epsilon^{\beta^{*}} \mathrm{H}_{\alpha \beta} \geq 0$, give

$$
\begin{aligned}
& 0 \leq \sigma_{s} \propto W_{2}\left(\frac{v}{-q^{2} m_{N}^{2}}+1\right)-W_{1} \Rightarrow \frac{F_{2}}{2 x}-F_{1} \geq 0 \\
& 0 \leq \sigma_{R}^{\sigma_{L}} \propto W_{1} \pm \frac{1}{2}\left(\frac{v^{2}}{m_{N}}-\frac{q^{2}}{m_{N}^{2}}\right)^{\frac{1}{2}} W_{3} \Rightarrow F_{1}+\frac{1}{2} F_{3} \geq 0 .
\end{aligned}
$$

Thus

$$
F_{2} \geq 2 x F_{1}, \quad F_{1} \geq \frac{1}{2}\left|F_{3}\right|
$$

Integrate over $\mathrm{y}$ :

$$
\begin{aligned}
\frac{\pi}{\mathrm{G}_{\mathrm{F}}^{2} \mathrm{~m}_{\mathrm{N}}^{\mathrm{E}} \nu} & \frac{\mathrm{d} \sigma}{\mathrm{dx}}\left(\frac{v}{v}\right)=\mathrm{a}_{\mathrm{S}}\left(\frac{v}{v}\right)+\left({ }_{\frac{1}{3}}^{1}\right) \times \mathrm{a}_{L}\left(\frac{v}{v}\right) \\
& +\left(\begin{array}{c}
\frac{1}{3} \\
{ }_{1}
\end{array}\right) \times \mathrm{xa}_{\mathrm{R}}\left(\frac{v}{v}\right)
\end{aligned}
$$

where $a_{S}=\frac{1}{2} F_{2}-x F_{1}, a_{L}=F_{1} \mp \frac{1}{2} F_{3}$.

4. Simple cons equences of scaling:

i) Adler sum rule

$$
\begin{aligned}
& \int_{1}^{\infty} \frac{\mathrm{d} \omega}{\omega}\left[F_{2}^{\bar{v}}-F_{2}^{v}\right]=\int_{0}^{1} \frac{\mathrm{dx}}{\mathrm{x}}\left[F_{2}^{\bar{v}}-F_{2}^{v}\right](\mathrm{x}) \\
& =<4 \mathrm{I}_{3} \cos ^{2} \theta_{c}+\left(3 y+2 I_{3}\right) \sin ^{2} \theta_{c}{ }^{>}{ }_{N} .
\end{aligned}
$$


In the scaling region, the left hand side is automatically $q^{2}$ independent.

ii) Total cross sections increase linearly with $\mathrm{E}_{v}$ :

$$
\sigma^{\nu, \bar{v}}=\mathrm{C}_{\nu, \bar{v}_{1}}
$$

where

$$
\begin{aligned}
& \mathrm{C}_{\nu, \bar{v}}=\frac{\mathrm{G}^{2} \mathrm{~m} \mathrm{~N}}{\pi} \int \mathrm{dx}\left[\mathrm{a}_{3}^{\nu, \bar{v}}(\mathrm{x})+\left(\begin{array}{c}
1 \\
\frac{1}{3}
\end{array}\right) \mathrm{xa}_{\mathrm{L}}^{\nu, \bar{v}}(\mathrm{x})+\left(\begin{array}{l}
\frac{1}{3} \\
\mathrm{x}^{2}
\end{array} \mathrm{a}_{\mathrm{R}}^{\nu, \bar{v}}(\mathrm{x})\right] .\right. \\
& \text { [Also }\left\langle\mathrm{q}^{2}>\propto v\right] \text {. }
\end{aligned}
$$

Experimentally

$$
c_{v} / c_{v}=1 / 3\left(\begin{array}{lll}
0.38 \pm 0.02 & \mathrm{CERN} \\
0.35 \pm 0.18 & \mathrm{NAL}
\end{array}\right)
$$

To understand this, set $\theta_{c}=0$ and consider an isosinglet target. From charge symmetry, get $F_{i} \nu p=F_{i}{ }^{\nu}, F_{i} \nu n=F_{i}^{\nu}$, or

$$
\begin{aligned}
& \mathrm{F}_{\mathrm{i}}{ }^{\nu}=\left(\mathrm{F}_{\mathrm{i}}{ }^{\nu \mathrm{p}}+\mathrm{F}_{\mathrm{i}}{ }^{\nu \mathrm{n}}\right) / 2=\left(\mathrm{F}_{\mathrm{i}} \bar{\nu} \mathrm{p}+\mathrm{F}_{\mathrm{i}} \bar{\nu} \mathrm{n}\right) / 2=\mathrm{F}_{\mathrm{i}}{ }^{\bar{v}}=\mathrm{F}_{\mathrm{i}} \\
& \frac{\sigma^{v}}{\sigma^{v}}=\frac{\int a_{s}+\frac{1}{3} \int x a_{L}+\int x a_{R}}{\int a_{S}+\int x a_{L}+\frac{1}{3} \int x a_{R}} .
\end{aligned}
$$

so

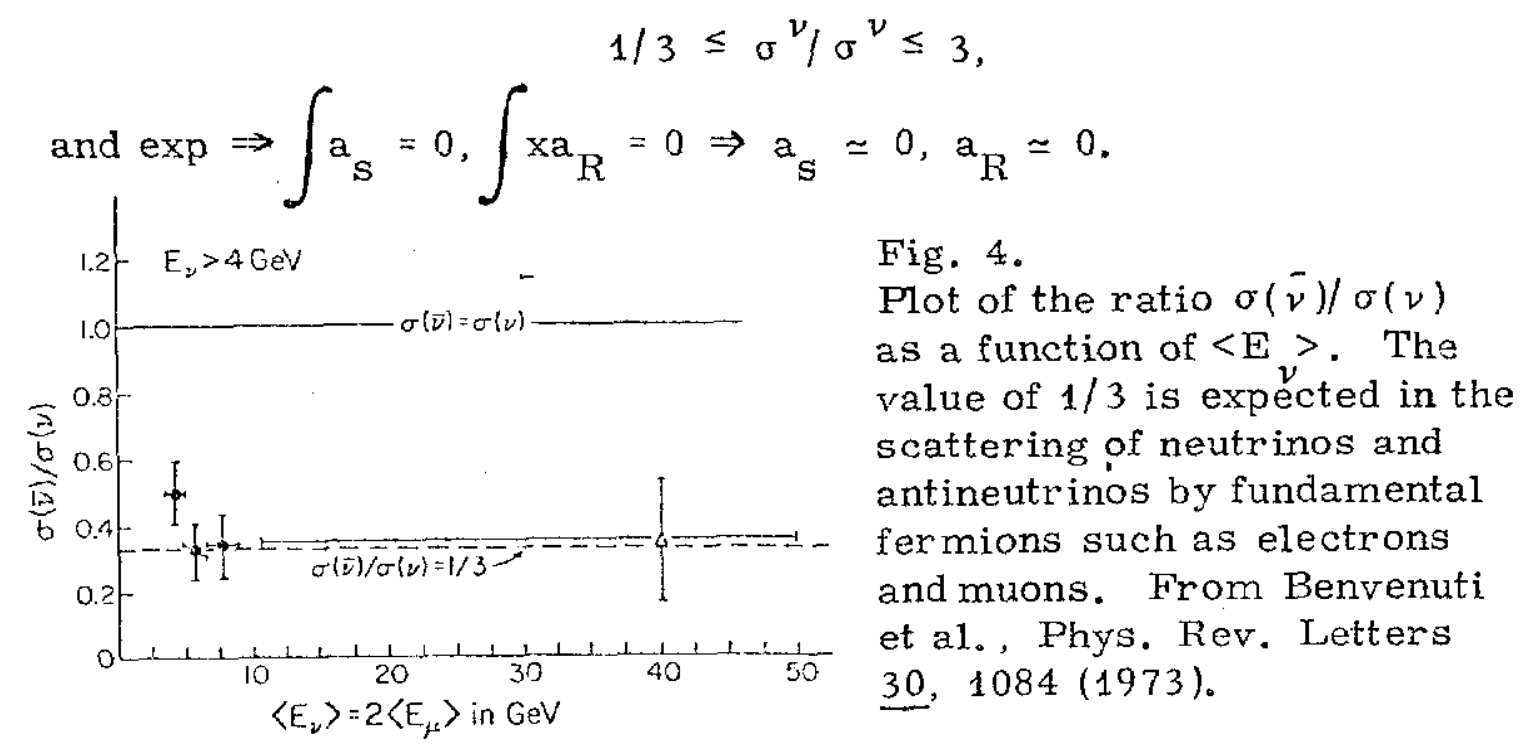




$$
\mathrm{a}_{\mathrm{S}} \simeq 0 \text { means } \mathrm{F}_{2} \simeq 2 \times \mathrm{F}_{1} .
$$

(Callan-Gross relation: spin 1/2 constituents)

$$
\begin{aligned}
& \mathrm{a}_{\mathrm{R}} \simeq 0 \text { means } \mathrm{F}_{3} \simeq-2 \mathrm{~F}_{1} \\
& \text { (Maximal VA interference). }
\end{aligned}
$$

iii) $y$-distribution; above considerations predict $y$-distribution.

$$
\begin{aligned}
& \frac{d^{2} \sigma}{d x d y}=\frac{G_{F}^{2} m_{N} E_{1}}{\pi} F_{2}(x) \text { for } v, \\
& =\frac{G_{F}^{2} m_{N} E_{1}}{\pi} F_{2}(x)(1-y)^{2} \text { for } \bar{v}
\end{aligned}
$$

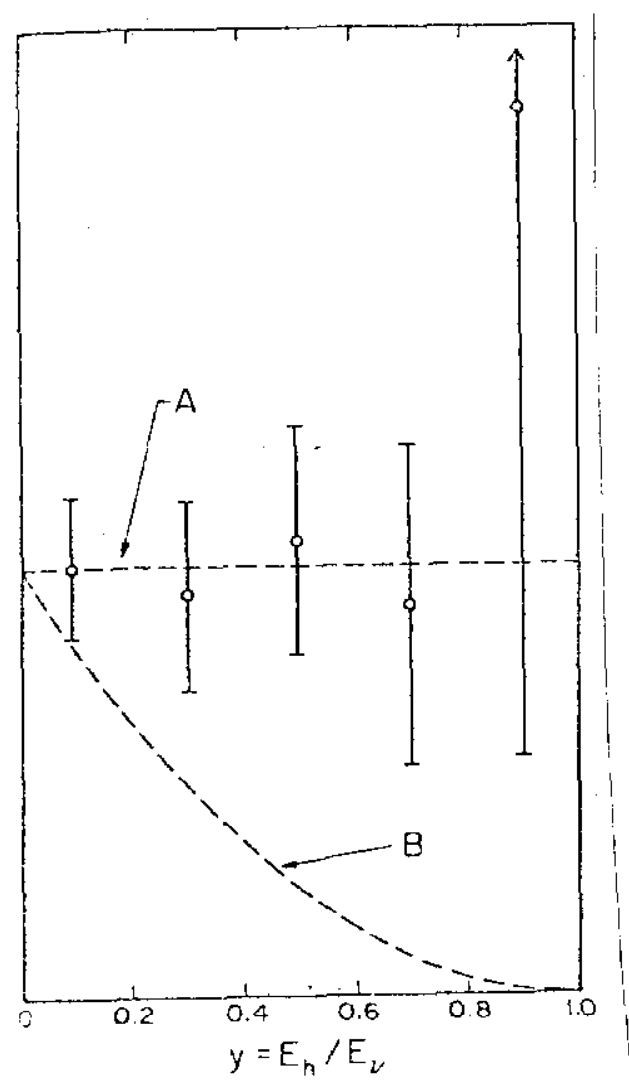

Fig. 5. $\mathrm{y}$-distribution in the inclusive $y$ process. From B.C. Barish et al., Phys. Rev. Letters 8, 565 (1973).

iv) Mean muon energy:

$$
\left\langle\underset{\mathrm{E}_{1}}{\mathrm{E}_{2}}\right\rangle=\langle 1-\mathrm{y}\rangle= \begin{cases}1 / 2 & \text { for } v \\ 3 / 4 & \text { for } \bar{v}\end{cases}
$$


This relation is flux independent.

v) Distribution in $z=x y$ :

$$
\begin{aligned}
& \frac{1}{N} \frac{d N}{d z}=\frac{d \sigma}{d z} \frac{1}{\sigma} \\
& =\frac{\int_{V}^{1} \frac{d x}{x} F_{2}(x)}{\int_{0}^{1} d x F_{2}(x)}
\end{aligned}
$$

This is flux, $v$ energy independent.

5. Regge Asymptotics.

From the Regge analysis of helicity amplitudes,

$$
\begin{aligned}
& W_{1} \longrightarrow{ }_{\nu \rightarrow \infty} \beta_{1}\left(q^{2}\right)_{v}^{\alpha_{1}(0)} \\
& W_{2} \rightarrow \beta_{2}\left(q^{2}\right) \nu_{2}(0)-2 \\
& W_{3} \longrightarrow \beta_{3}\left(q^{2}\right) v^{\alpha_{3}(0)-1} \quad G=-1(\omega, \phi): \alpha_{3}(0) \simeq 1 / 2 .
\end{aligned}
$$

If Regge and Bjorken limits are simultaneously valid, $\beta_{i}$ must be
power behaved:

and

$$
\beta_{1,3} \sim\left(\frac{1}{q^{2}}\right)^{\alpha_{1}, \alpha_{3}}, \quad \beta_{2} \sim\left(\frac{1}{q^{2}}\right)^{\alpha_{2}-1}
$$

$$
\begin{gathered}
\mathrm{F}_{1} \rightarrow \beta_{1} \omega_{1}^{\alpha_{1}(0)}, \\
F_{2} \rightarrow \beta_{2} \omega_{1}^{\alpha_{1}(0)-1}: \text { const as } \omega \rightarrow \infty, \\
F_{3} \rightarrow \beta_{3} \omega^{\alpha_{3}(0) .} .
\end{gathered}
$$

Also 


$$
F{ }_{2}^{\nu p}-F_{2}^{\overline{\nu p}} \sim \omega^{\alpha} \rho^{(0)-1} \sim \frac{1}{\sqrt{\omega}} \text { as } \omega \rightarrow \infty \text {, etc. }
$$

IV. SCALING AND QUARK PARTON MODEL

1. Assumptions: Infinite momentum frame description of hadrons in terms of almost free constituents of light mass.

i) Leptons scatter incoherently from partons.

ii) Partons are almost free: They are near their mass shell before and after interaction.

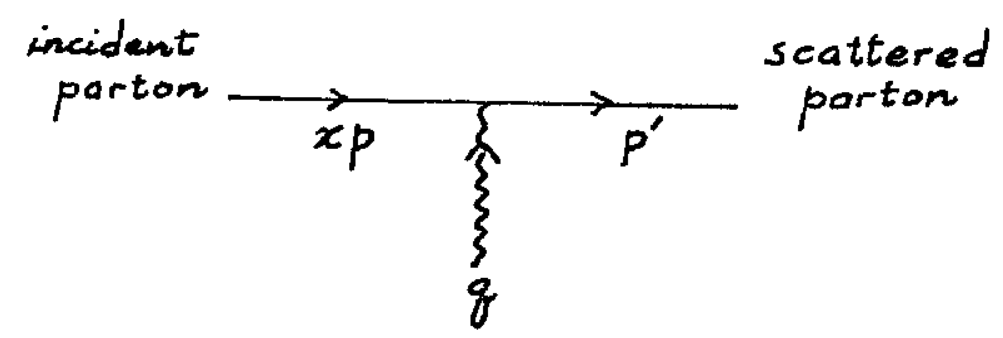

Fig. 6.

Parton kinematics. $\mathrm{x}$ is the fraction of longitudinal momentum carried by parton.

$$
0 \simeq\left(m_{\text {parton }}\right)^{2}=p^{\prime 2}=(x p+q)^{2} \simeq 2 x p \cdot q+q^{2} \text {, }
$$

or

$$
x \simeq-q^{2} / 2 p \cdot q
$$

i.e., for given $\mathrm{q}^{2}$ and $\nu$, deep inelastic scattering probes the parton distribution with longitudinal momentum fraction $x$. [References: R. P. Feynman, "Hadron-Photon Interactions", (Benjamin, NY., (1972); D. H. Perkins, in Proceedings of Chicago-Batavia Conference (1972), Vol. 4, p. 189.]

2. Structure function in the parton model.

$$
H_{\alpha \beta}^{v, \bar{v}}=\sum_{\mathrm{i}} \mathrm{u}_{\mathrm{i}}(\mathrm{x}) \mathrm{h}_{\alpha \beta}^{\nu, \bar{v}}
$$
$u_{i}(x)$ : probability that a parton (anti-parton) of type $i$ be found
with $x$.

$\mathrm{h}_{\alpha \beta}$ : structure function for a parton calculated in the Born approximation. 


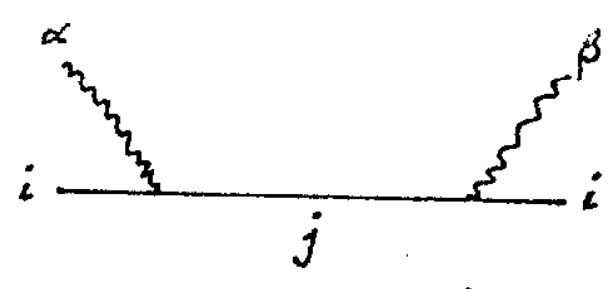

Fig. 7

Born diagram for the parton structure function $\mathrm{h}_{\alpha \beta}$.

$$
J_{\mu}=\cos \theta_{c} \frac{\bar{p} \gamma_{\mu}\left(1-\gamma_{5}\right) n}{\text { pure } V-A}
$$

$$
\begin{aligned}
& h_{\alpha \beta}=-x g_{\alpha \beta}+2 x^{2} \frac{p_{\alpha} p_{\beta}}{p \cdot q} \pm i x \frac{\epsilon_{\alpha \beta \rho \sigma} p^{\rho_{q}^{\sigma}}}{p \cdot q} \text { for } \begin{array}{l}
\text { parton } \\
\text { anti-parton }
\end{array} \\
& =-g_{\alpha \beta} \omega_{1}+\frac{p_{\alpha} p_{\beta}}{m_{N}^{2}} \omega_{2}-i \epsilon_{\alpha \beta \rho \sigma} \frac{p^{\rho} q^{\sigma}}{2 m_{N}^{2}} \omega_{3} .
\end{aligned}
$$

So

$$
\left.\begin{array}{rl}
f_{1}=\omega_{1}=x & f_{2}=p \cdot q \omega_{2} / m_{N}^{2}=2 x^{2} \\
f_{3}=p \cdot q \omega_{3} / m_{N}^{2}=\mp 2 x
\end{array}\right\} \begin{aligned}
f_{2} & =2 x f_{1}=F_{2}-2 x F_{1} \\
& \text { (Callan-Gross) } \\
f_{3} & =-2 f_{1} \text { (partons) } \\
= & +2 f_{1} \text { (antipartons) }
\end{aligned}
$$

Experimentally $F_{3} \simeq-2 F_{1} \Rightarrow u_{p}, u_{n}, u_{-\lambda}=0$

With Callan-Gross relation we can write.

$$
\begin{gathered}
\frac{d^{2} \sigma}{d x d y}=\frac{G_{F^{2}}^{2} m_{N} E_{1}}{\pi} \times\left\{\left[\begin{array}{c}
1 \\
(1-y)^{2}
\end{array}\right] F_{L}(x)+\left[\begin{array}{c}
(1-y)^{2} \\
1
\end{array}\right] F_{R}(x)\right\} \\
\text { for }\left(\begin{array}{c}
\nu \\
v
\end{array}\right)
\end{gathered}
$$

where

$$
F_{L}^{k}=\sum_{i=p, n, \lambda} a_{i}^{k} u_{i}(x),
$$




$$
F_{R}^{k}=\sum_{i=\bar{p}, \bar{n}, \bar{\lambda}} b_{i}^{k} \ddot{u}_{i}(x),
$$

$\mathrm{k}$ denotes different processes, $\nu p, \bar{\nu}, \bar{\nu}, \nu \mathrm{n}$, ep, and en.

3. Equality: From the above linear relations follow

$$
12\left(F_{1}^{e p}-F_{1}^{e n}\right)=F_{3}^{\nu p}-F_{3}^{\nu n} \text { (not tested) }
$$

4. Sum Rules:

$$
\begin{gathered}
<S>=\int_{0}^{1} d x\left[u_{\lambda}-u_{\bar{\lambda}}\right] \\
<I_{3}>=\int_{0}^{1} d x \frac{1}{2}\left[u_{p}-u_{\bar{p}}-u_{n}+u_{\bar{n}}\right] \\
<B>=\int_{0}^{1} d x \frac{1}{3}\left[u_{p}+u_{n}+u_{\lambda}-u_{p}-u_{n}-u_{\lambda}\right]
\end{gathered}
$$

From these

a. Adler sum rule $\leqslant I_{3}$. $\infty$

b. Llewellyn Smith-Gross: $-\int \frac{d \omega}{\omega}\left(F_{3}^{v}+F_{3}{ }_{3}\right)=$

$$
=<4 B+Y\left(2-3 \sin ^{2} \theta_{c}\right)+2 I_{3} \sin ^{2} \theta_{c}>\text {. }
$$

5. Inequalities: $u_{i} \geq 0$.

$$
F_{2}^{e p}+F_{2}^{\text {en }}-\frac{5}{18}\left(F_{2}^{\nu p}+F_{2}^{\nu n}\right) \propto \text { pos. const }\left(u_{\lambda}+u_{\lambda}\right) \geq 0 \text {. }
$$

Experimentally

$$
\int_{0}^{1} \mathrm{dx}\left(\mathrm{F}_{2}^{\mathrm{ep}}+\mathrm{F}_{2}^{\mathrm{en}}\right) \simeq \frac{5}{18} \int_{0}^{1} \mathrm{dx}\left(\mathrm{F}_{2}^{\nu \mathrm{p}}+\mathrm{F}_{2}^{\nu \mathrm{n}}\right)
$$

implying $u_{\lambda} \approx u_{\lambda} \approx 0$, and $\frac{18}{5} F_{2}^{e d}(x) \simeq F_{2}^{\nu d}(x)$.

This is a test for fractionally charged quark partons. 


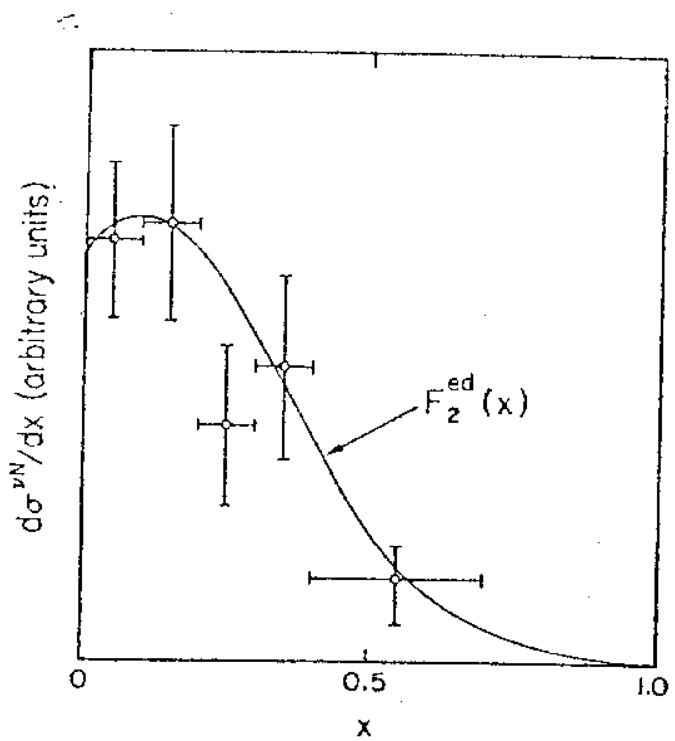

Fig. 8

The distribution $\mathrm{d} \sigma^{\nu \mathrm{N}} / \mathrm{dx}$

(arbitrary units) versus $\mathrm{x}=\mathrm{Q}^{2} / 2 \mathrm{~m} \nu$ (the scaling variable). For comparison, the fit for $F_{2}^{e d}(x)$ is shown. From B. C. Barish et al., Phys. Rev. Letters 8, 565 (1973). See also F. Sciulli's contribution.

6. Overall picture

$$
\frac{d \sigma}{d x d y}=\frac{G_{F}^{2} m_{N} E^{2}}{\pi} 2 x \begin{cases}u_{n}(x) & v p \\ (1-y)^{2} u_{p}(x) & \overline{v p} \\ u_{p}(x) & v n \\ (1-y)^{2} u_{n}(x) & \bar{v} n\end{cases}
$$

\section{SCALING AND LIGHT CONE}

1. Light Cone Analysis

$$
\begin{aligned}
& T_{\mu \nu}(p, q)=\int d^{4} x e^{i q \cdot x}<p\left|\left[j_{\mu}\left(\frac{x}{2}\right), j_{\nu}^{\dagger}\left(-\frac{x}{2}\right)\right]\right| p> \\
& =\int d^{2} x_{\perp} \int d t d z e^{i \frac{\nu}{m}(t-z)-i m x(t+z)}<p|[]| p>
\end{aligned}
$$

Dominant contribution comes from $x^{2} \simeq 0$, because $t-z \simeq 0\left(\frac{m}{v}\right)$ and because of the commutativity of two currents at space-like separation.

Wilson's operator product expansion [K. Wilson, Phys. Rev. $179,1499(1969)]$. 


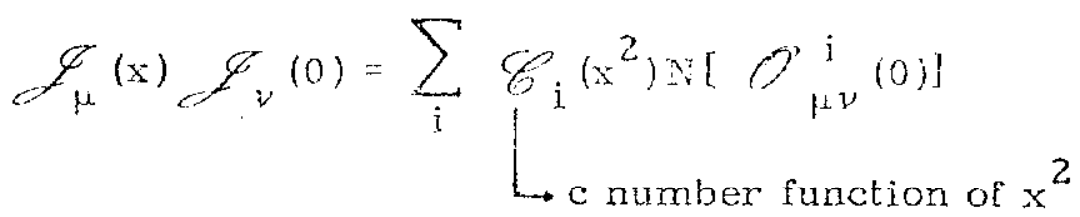

Gell-Mann, Fritsche postulate that leading light cone singularity structure of $\mathscr{F}_{\mu}(\mathrm{x}) \mathscr{F}_{\nu}^{\dagger}(0)$ same as that of the free quark model $\rightarrow$ reproduce predictions of the quark parton model, and scaling. [ See, for example, Gross and Treiman, Phys. Rev. D4, 1059 (1971).]

More rigorously [see, for example, D. Gross, "Scaling in Quantum Field Theory, "unpublished],

$$
\begin{aligned}
\mathrm{T}\left[\mathrm{J}_{\alpha} \frac{\mathrm{x}}{2} \mathrm{~J}_{\beta}-\frac{\mathrm{x}}{2}\right] & =\sum_{\mathrm{n}=0}^{\infty} \mathscr{C}^{(\mathrm{n})}\left(\mathrm{x}^{2}\right) \vartheta_{\alpha \mu_{1} \ldots \mu_{\mathrm{n}}}(0) \mathrm{x}^{\mu_{1}} \ldots \mathrm{x}^{\mu_{\mathrm{n}}} \\
& + \text { terms contributing to } \mathrm{W}_{1}, \mathrm{~W}_{3} \\
& + \text { operators of "twist" }>2,
\end{aligned}
$$

where

$$
\begin{aligned}
& O_{\alpha \beta_{1}} \ldots \mu_{n} \simeq \bar{\psi} \gamma_{\alpha} \partial_{\beta} \gamma_{\mu_{1}} \cdots \psi \text {, etc. } \\
& \text { sym. and traceless in } \alpha \beta, \mu_{1}, \ldots \mu_{n} \\
& \text { twist }=\text { dimensions - spin } \\
&=(3+n+1)-(n+2)=2 .
\end{aligned}
$$

Twist $>2$ operators contribute to $W^{t}$ s asymptotically down by full powers of $\left(\mathrm{q}^{2}\right)^{-1}$.

Take the matrix element of $\mathcal{O}_{\alpha \beta} \ldots$ and spin average:

$$
\bar{\Sigma}<p\left|O_{\alpha \beta \mu_{1} \ldots \mu_{n}}(0)\right| p>\sim p_{\alpha} p_{\beta} p_{\mu_{1}} \ldots p_{\mu_{n}} \cdot
$$

The $n$-th term contains $n+2$ factors of $p$. Compare this to the forward Compton amplitude:

$$
p_{\alpha} p_{\beta} \int \mathrm{d} v^{\prime} \frac{W_{2}\left(v^{\prime}, q^{2}\right)}{v^{\dagger}-v} \cong p_{\alpha} p_{\beta} \sum_{n}(p \cdot q)^{n} \int d x x^{\dot{n}} \cdot\left[v^{\prime} W_{2}\left(v, q^{2}\right)\right]
$$

Thus 


$$
\begin{gathered}
\int_{0}^{1} d x x^{N}\left[\frac{\nu W_{2}\left(\nu, q^{2}\right)}{m_{N}^{2}}\right]=C^{(N)}\left(q^{2}\right) \\
\widetilde{C}^{(N)}\left(q^{2}\right) \simeq\left(q^{2}\right)^{n+1}\left(\frac{\partial}{\partial q^{2}}\right)^{n} \int d^{4} x e^{i q \cdot x} \mathscr{C}^{n}\left(x^{2}\right)
\end{gathered}
$$
For free fields, $\widetilde{C}^{(N)}\left(q^{2}\right)$ is independent of $q^{2} \Rightarrow \nu W_{2}\left(\nu, q^{2}\right)$ a
function only of $x$.

2. Interacting Fields and Renormalization Groups.

Coefficient functions $\widetilde{\mathrm{C}}^{(\mathrm{N}) \mathrm{i}}\left(\mathrm{q}^{2}\right)$ satisfy the renormalization group equation .

$$
\left[\mu \frac{\partial}{\partial \mu}+\beta(g) \frac{\partial}{\partial g}-\gamma^{(N) i}(g)\right] C_{A S}^{(N) i}\left(q^{2} / \mu^{2}, g\right)=0 .
$$

The solution is of the form

$$
C^{(N) i}\left(q^{2} / \mu^{2}, g\right) \underset{-q^{2} \rightarrow \infty}{\longrightarrow} C^{(N) i}\left(-q^{2}\right)^{-\frac{1}{2} \gamma}{ }^{(N) i}\left(g_{\infty}\right)
$$

where $g_{\infty}$ is a root of $\beta(g)$ :

$$
\beta\left(g_{\infty}\right)=0 \text { and } d \beta(g) /\left.d g\right|_{g=g_{\infty}}<0
$$

and $\gamma^{(N) i}$ is the anomalous dimension of $\mathcal{O}^{(N) i}, \gamma^{(N) i}(g=0)=0$; increases with $\mathrm{N}$ (positivity of $\nu \mathrm{W}_{2}$ ) and is convex downwards $\left(\rightarrow 2 \gamma_{\psi}\right.$ as $\left.n \rightarrow \infty\right)$.

Consider $\mathcal{O}^{(\mathrm{N}) \mathrm{i}}=\Theta_{\mu \nu} ;$ conserved energy momentum tensor $(\mathrm{D}=4, \mathrm{~J}=2$, twist 2$)$. $\Theta_{\mu \nu}$ has no anomalous dimensions, $\gamma^{\Theta}=0$. Now moment $x^{N} \leftrightarrow \operatorname{spin} N+2$ operators.

$$
\int_{0}^{1} \mathrm{dx} \nu \mathrm{W}_{2}=\text { const }+\frac{\mathrm{C}}{|q|^{\nu}} .
$$

$\Theta_{\mu \nu}$ is isoscalar $\Rightarrow$ 


$$
\int_{0}^{1} \mathrm{dx} v \mathrm{w}_{2}^{\nu p}\left(\nu, q^{2}\right)=\int_{0}^{1} \nu \mathrm{W}_{2}^{\nu n}\left(\nu, q^{2}\right) \text { as }-q^{2} \rightarrow \infty
$$

or, $\left(\right.$ with $\left.\theta_{c} \rightarrow 0\right)$

$$
\int_{0}^{1} \mathrm{dx}\left[v \mathrm{w}_{2}^{\nu \mathrm{p}}-\nu \mathrm{W}_{2}^{\bar{\nu} \mathrm{p}}\right] \rightarrow 0
$$

We need consider two cases. [See, for example, S. Adler, NAL-Conf-74/39-THY] :

(1) $g_{\infty} \neq 0 . \quad \gamma^{(N)}\left(g_{\infty}\right) \neq 0$ in general $\Rightarrow$ moments go to zero as $\left(-q^{2}\right)^{-\frac{1}{2} \gamma^{(N)}}$

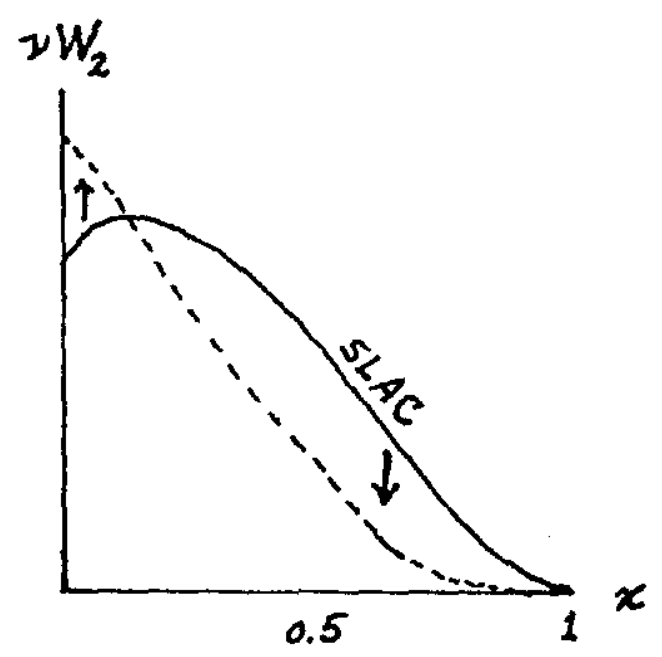

Fig. 9

Behavior of $v W_{2}$ as $q^{2} \rightarrow \infty$ as predicted by field theories (schematic).

$\nu W_{2}$ will change as $-q^{2} \rightarrow \infty$. (a) Near $x=1$, decreases to make higher moments go to zero; (b) If $y^{(2) i}$ all small, area remains = const; (c) near $x \simeq 0$ increases.

(2) $g_{\infty}=0$ (Asymptotic freedom) 


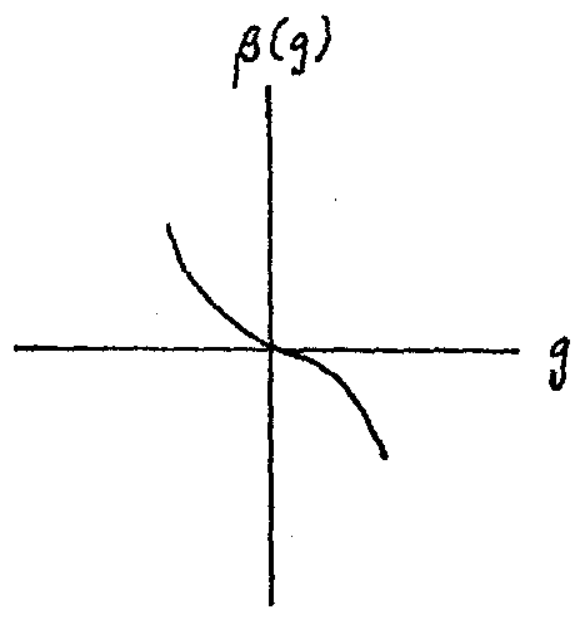

Fig. 10 .

Behavior of $\beta(\mathrm{g})$ in asymptotically free gauge theory of strong interactions.

This happens in nonabelian gauge theories.

$$
C^{(N) i}\left(q^{2}\right) \underset{-q^{2} \rightarrow \infty}{\rightarrow}\left(\ln -q^{2}\right)^{-\frac{1}{2} a^{(N) i}}
$$

where $a^{(N) i}$ are computable numbers, independent of g. Approach to as ymptotic logarithmic; $\nu W_{2}$ behavior as $-q^{2} \rightarrow \infty$ similar to above; since $a_{(n) i}$ known, possible to extrapolate $W_{2}$ from $\left(x,-q^{2}\right)$ to $\left(x,-q^{2}\right)$. Combined with the Bloom-Gilman hypothesis, gives bounds on e.m. form factors. For implications of asymptotic freedom on neutrino physics, see the contribution of A. Zee.

3. Remarks

A. If exact scaling remains valid, all known field theory models of strong interactions in trouble.

B. Parton model works at SLAC and NAL v energies, whether you believe in it or not--a case of Niels Bohr's horseshoe.

C. If scaling breaks down as field theory predicts, $\sigma_{\nu}-\sigma_{\nu} \rightarrow 0$. Adler sum rule OK, but not Llewellyn Smith-Gross.

\section{GAUGE THEORIES}

1. Gauge Theories of Weak Interactions.

General References:

B. W. Lee, Proceedings of Chicago-Batavia Conference, (1972), Vol. 4, p. 249.

S. Weinberg, Proceedings of Aix-en-Provence Conference

(1973); Rev. Modern Physics, to be published.

i) Unification of electromagnetic and weak interactions in a 
gauge theory (nonabelian).

ii) Spontaneously Broken Gauge Symmetry: masses of weak bosons arise thru this mechanism. The symmetry breaking is especially "soft" $\rightarrow$ The renormalizability of the unbroken gauge theory is preserved.

In a renormalizable theory, bad high energy behavior of a tree graph has to be cancelled by another. Example:

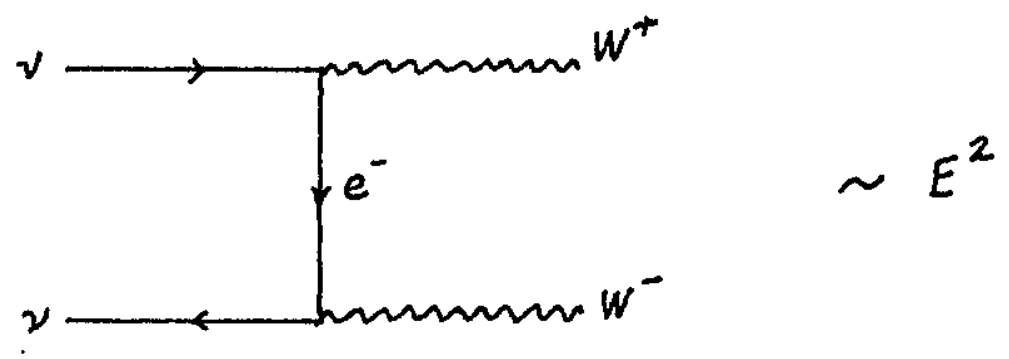

Fig. 11.

The process $v+\bar{v} \rightarrow \mathrm{W}^{+}+\mathrm{W}^{-}$in the conventional theory.

The $\mathrm{E}^{2}$ - E-terms must be cancelled by one or the other of the following graphs:
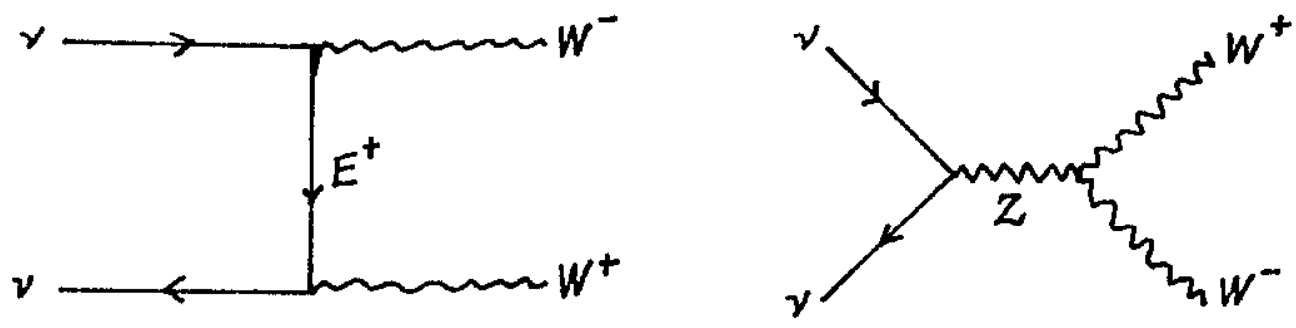

Fig. 12

Two tree diagrams for $v+\vec{v} \rightarrow \mathrm{W}^{+}+\mathrm{W}^{-}$that appear in a renormalizable theory.

Because gauge theories are renormalizable, they must contain either heavy leptons, neutral currents or both.

iii) Important Issues:

Heavy leptons

Neutral currents

Charms

Nuclear physics

2. Neutral Current in the Weinberg-Salam Theory. 


$$
Q=T_{3}+Y / 2
$$

Example: $\quad\left(\begin{array}{l}\nu \\ \text { e }\end{array}\right)_{\mathrm{L}}, \quad T=1 / 2, \quad Y=-1$

$$
e_{R}, T=0, Y=-2 .
$$

Neutral current-gauge boson coupling:

$$
\mathscr{L}=\mathrm{g} \mathrm{j}_{\mu}^{3} \mathrm{~A}^{3 \mu}+\mathrm{g}^{\mathrm{e}} \frac{\left(\mathrm{j}_{\mu}^{\mathrm{em}}-\mathrm{j}_{\mu}^{3}\right) \mathrm{B}^{\mu} .}{\frac{1}{2} \times{ }^{\text {"hypercharge" current }}}
$$

Let

$$
\left(\begin{array}{c}
A_{\mu}^{3} \\
B
\end{array}\right)=\left(\begin{array}{cc}
\cos \theta_{W^{\prime}} & \sin \theta_{W} \\
-\sin \theta_{W}, & \cos \theta_{W}
\end{array}\right)\left(\begin{array}{c}
Z_{\mu} \\
A_{\mu}
\end{array}\right) \text { massive }
$$

Then

with

$$
\mathscr{L}=e j_{\mu}^{e m} A^{\mu}+\sqrt{g^{2}+g^{12}}\left[j_{\mu}^{3}-\sin ^{2} \theta_{W}{ }_{\mu}^{e m}\right] z^{\mu}
$$

$$
\begin{gathered}
e=g g^{\prime}\left(g^{2}+g^{\prime 2}\right)^{-1 / 2} \\
\cos \theta_{W}=\frac{g}{\sqrt{g^{2}+g^{\prime 2}}} \cdot \\
\mathscr{L}_{Z}=\sqrt{g^{2}+g^{\prime 2}} Z^{\mu}\left[J_{\mu}^{3}-\sin \theta_{W} J_{\mu}^{e m}+\frac{1}{2} \bar{\nu}_{\mu}\left(1-\gamma_{5}\right) \nu+\ldots\right]
\end{gathered}
$$

Effective Local Coupling:

$$
\frac{g^{2}+g^{\prime 2}}{8 m_{z}^{2}} \bar{v} \gamma^{\mu}\left(1-\gamma_{5}\right) \nu[\underbrace{\text { e. m. current }}_{\substack{\text { isospin rotation } \\ \rightarrow \text { charged current }}}
$$

In the original construction of Weinberg and Salam, a particularly 
simple symmetry breaking mechanism was used, leading to the relation

$$
\frac{m_{Z}^{2}}{m_{W}^{2}}=\frac{g^{2}+g^{2}}{g^{2}} \text {, or } \frac{g^{2}+g^{2}}{m_{Z}^{2}}=\frac{g^{2}}{m_{W}^{2}}
$$

In general, however, there need not be any relation between ${ }^{m} \mathrm{~W}$ and $\mathrm{m}_{\mathrm{Z}}$ : Parameterize the strength of $\mathrm{Z}$ exchange by one parameter $\mathrm{x}$ :

$$
\begin{gathered}
\frac{g^{2}+g^{\prime 2}}{m_{Z}^{2}}=x \frac{g^{2}}{m_{W}^{2}} . \\
\Rightarrow \frac{G_{F}}{\sqrt{2}} \times \nu \gamma^{\mu}\left(1-\gamma_{5}\right) \nu\left[V_{\mu}^{3}-A_{\mu}^{3}-2 \sin ^{2} \theta_{W} J_{\mu}^{e m}\right] .
\end{gathered}
$$

3. Tests of the WS Theory in Hadronic Reactions.

While the properties of neutral current ought to be studied in the most general context, we will consider parameterizing neutral current effects a'la Weinberg-Salam theory.

i) Inclusive Reactions: Define

$$
\mathrm{R}_{\nu}=\sigma\left(\bar{\nu}_{\mu}^{\nu_{\mu}}+\mathrm{N} \rightarrow \bar{\nu}_{\mu}^{v_{\mu}}+\mathrm{B}\right) / \sigma\left(\bar{\nu}_{\mu}^{\nu_{\mu}}+\mathrm{N} \rightarrow{ }_{\mu}^{+}+\mathrm{B}\right) .
$$

(A) The hadronic neutral current is in the quark language $\left(\right.$ with $\left.\theta_{c}=0\right)$ :

$$
\begin{aligned}
& \overline{\mathrm{p}} \gamma_{\mu}\left[\mathrm{a}\left(1-\gamma_{5}\right)+\mathrm{b}\left(1+\gamma_{5}\right)\right] \mathrm{p} \\
& +\overline{\mathrm{n}} \gamma_{\mu}\left[\mathrm{c}\left(1-\gamma_{5}\right)+\mathrm{d}\left(1+\gamma_{5}\right)\right] \mathrm{n}
\end{aligned}
$$

where

$$
\begin{gathered}
a=1 / 2-(2 / 3) \sin ^{2} \theta_{W}, b=-(2 / 3) \sin ^{2} \theta_{W} \\
c=-1 / 2+(1 / 3) \sin ^{2} \theta_{W}, b=(1 / 3) \sin ^{2} \theta_{W} \\
R_{V}=\frac{1}{2}-\sin ^{2} \theta_{W}+\frac{20}{27} \sin ^{4} \theta_{W}
\end{gathered}
$$




$$
R_{\nu}=\frac{1}{2}-\sin ^{2} \theta_{W}+\frac{20}{9} \sin ^{4} \theta_{W} .
$$

[ A. De Rujula et al. , Revs. Mod. Phys., to be published.]

(B) Assuming scaling in electroproduction,

$$
R_{\nu} \geq \frac{1}{2}\left\{1-2 \sin ^{2} \theta_{W} \sqrt{t}\right\}^{2}
$$

Assuming scaling both in eN and $v N(\bar{v} \mathrm{~N})$,

$$
R_{v} \geq \frac{1}{2}\left\{\frac{2}{3}+\frac{1}{3} x-\left(1-x^{2}\right) t\right\}
$$

where

$$
t=\frac{G^{2}}{\pi} \frac{4}{3} m_{N} E_{1} \int_{0}^{1} d x \cdot F_{2}^{e N}(x) / \sigma\left(\nu_{\mu}+N \rightarrow \bar{\mu}+B\right)
$$

Using

$$
\begin{gathered}
\int_{0}^{1} \mathrm{dx} \mathrm{F}_{2}^{\mathrm{eN}}(\mathrm{x}) \simeq 0.14, \sigma\left(v_{\mu}+\mathrm{N} \rightarrow \bar{\mu}+\mathrm{B}\right) \\
\frac{\mathrm{G}^{2}}{\pi} \mathrm{m}_{\mathrm{N}} \mathrm{E}_{1}, 0.52,
\end{gathered}
$$

one gets $t \cong 0.36$.

Take $t=1 / 3$. One gets

$$
\begin{aligned}
& R_{v}=\frac{1}{2} \cdot \frac{1}{3}\left(1+x+x^{2}\right), \\
& R_{v}=\frac{1}{2}\left(1-x+x^{2}\right)
\end{aligned}
$$

[ Pais and Treiman, Phys. Rev. D6, 2700 (1972); Paschos and Wofenstein, Phys. Rev. D7, $91(1973)]$.

ii) Exclusive Reactions

(A) Elastic: Theory predicts

$$
0.15 \leq \sigma(\nu+p \rightarrow \nu+p) / \sigma(\nu+n \rightarrow \mu+p)
$$




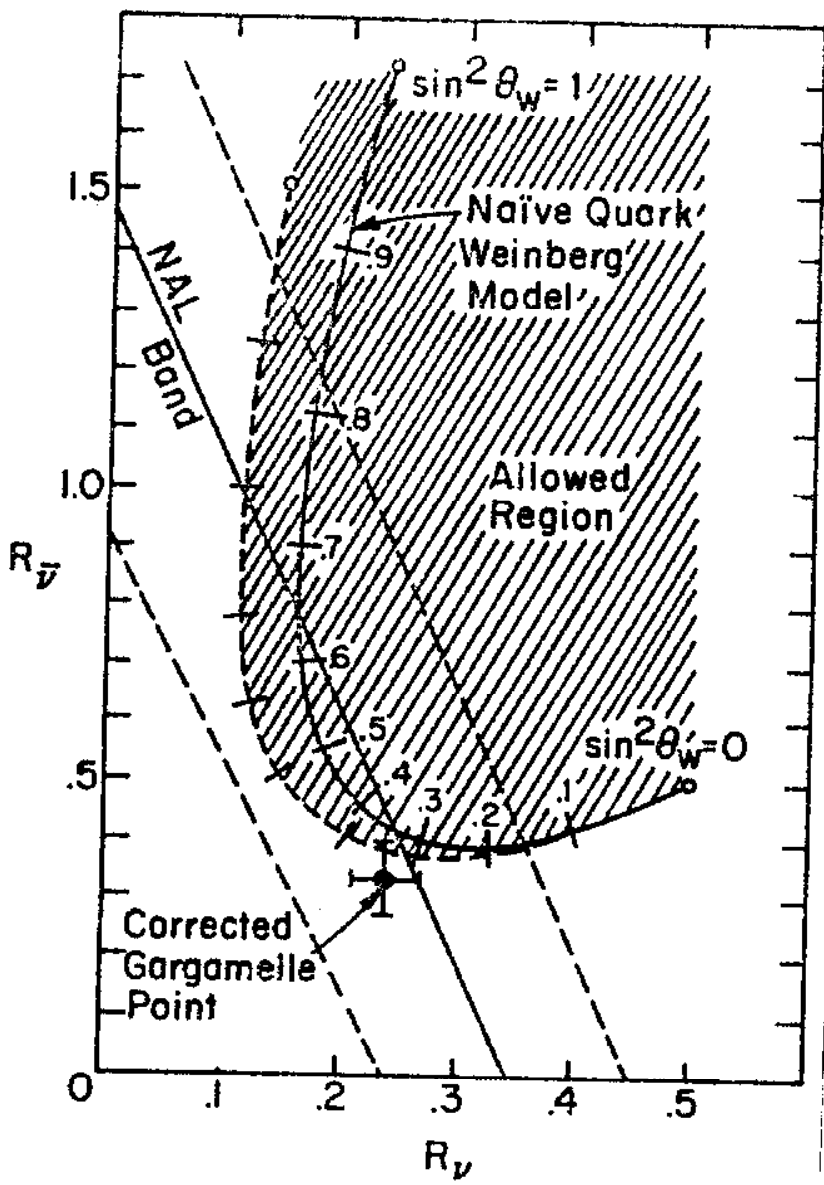

Fig. 13.

Comparison of theory and experiment for processes $(v, \bar{v})+N \rightarrow(v+\bar{v})+$ anything. From A. De Rujula et al., Revs. Mod. Phys., to be published.

$$
\leq 0.25 \text { for } \sin ^{2} \theta \leq 1 / 2
$$

Experiment (Gargamelle) gives $0.12 \pm 0.06$

(B) $\triangle$ Production. Define

$$
R=\frac{\sigma\left(v_{\mu}+p \rightarrow v_{\mu}+p+\pi^{0}\right)+\sigma\left(\nu_{\mu}+n \rightarrow \nu_{\mu}+n+\pi^{0}\right)}{2 \sigma\left(\nu_{\mu}+n \rightarrow \mu^{-}+p+\pi^{0}\right)} .
$$

$\triangle$ dominance gives $R \geq 0.4 \sim 0.5$ for $\sin ^{2} \theta_{W}<1 / 3$.

Refined theory (Adler, Nussinov, and Paschos, Phys. Rev. D, to be published) gives

$\begin{array}{llcc}\sin ^{2}{ }{ }_{W} & \text { s only } & +I=1 / 2 & \text { t.charge exch. in Al } \\ 0.3 & 0.56 & 0.40 & 0.23 \\ 0.4 & 0.46 & 0.33 & 0.18\end{array}$


Experiment (W. Lee-Columbia) gives $R<0.14$ (90\% CL). [W. Lee, Phys. Letters 40B, 423 (1972)I。

4. Tests of the WS Theory in Leptonic Processes.

$$
\begin{aligned}
& \mathrm{H}_{W}(v e \rightarrow \mathrm{e} v)=\frac{\mathrm{G}}{\sqrt{2}} \bar{\nu}_{\mu} \gamma^{\alpha}\left(1-\gamma_{5}\right) \nu_{\mu} \overline{\mathrm{e}}_{\alpha}\left(\mathrm{g}_{\mathrm{V}}-\mathrm{g}_{\mathrm{A}} \gamma_{5}\right) \mathrm{e} \\
& +\bar{\nu}_{\mathrm{e}} \gamma^{\alpha}\left(1-\gamma_{5}\right) \nu_{\mathrm{e}} \overline{\mathrm{e}}_{\alpha}\left(\mathrm{G}_{\mathrm{V}}-\mathrm{G}_{\mathrm{A}} \gamma_{5}\right) \mathrm{e}
\end{aligned}
$$

where

$$
\begin{aligned}
& \mathrm{G}_{\mathrm{A}}=1+\mathrm{g}_{\mathrm{A}} \\
& \stackrel{L_{\mathrm{V}}}{\mathrm{g} \text { cheutral current }}
\end{aligned}
$$
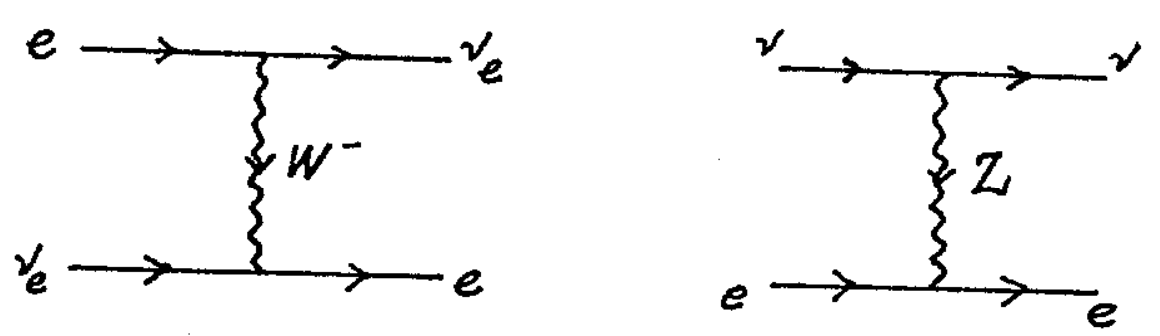

Fig. 14

Tree diagrams for $v+\mathrm{e} \rightarrow v+\mathrm{e}$.

$$
\begin{gathered}
g_{V}=\left(-\frac{1}{2}+2 \sin ^{2} \theta_{W}\right) x \\
g_{A}=\left(-\frac{1}{2}\right) x_{0}
\end{gathered}
$$

(A) $\sigma\left(\bar{v}_{\mathrm{e}}+\mathrm{e} \rightarrow \bar{v}_{\mathrm{e}}+\mathrm{e}\right)=\mathrm{C} 10^{-41} \mathrm{~cm}^{2}(\mathrm{E} / \mathrm{GeV})$.

$$
\begin{aligned}
C & =0.54 \text { in standard } V-A \\
& =0.136 \sim 2.86 \text { in WS }
\end{aligned}
$$
find

Gurr, Reines and Sobel, Phys. Rev. Letters 228, 1406 (1972)

$$
\sigma \leq 3 \sigma_{\mathrm{V}-\mathrm{A}}(90 \% \mathrm{cL})
$$


(B) Gargamelle had 370,000 pix each for $\nu_{\mu}$ and $\bar{v}_{\mu}$.

$\begin{array}{lccc} & \text { WS } & \text { background (est) } & \text { observed } \\ v_{\mu} & 0.6 \sim 6.0 & 0.3 \pm 0.2 & 0 \\ \bar{v}_{\mu} & 0.4 \sim 8.0 & 0.03 \pm 0.02 & 1\end{array}$

[F.J. Hassert et a1., Phys. Letters 46B, 121 (1973)].

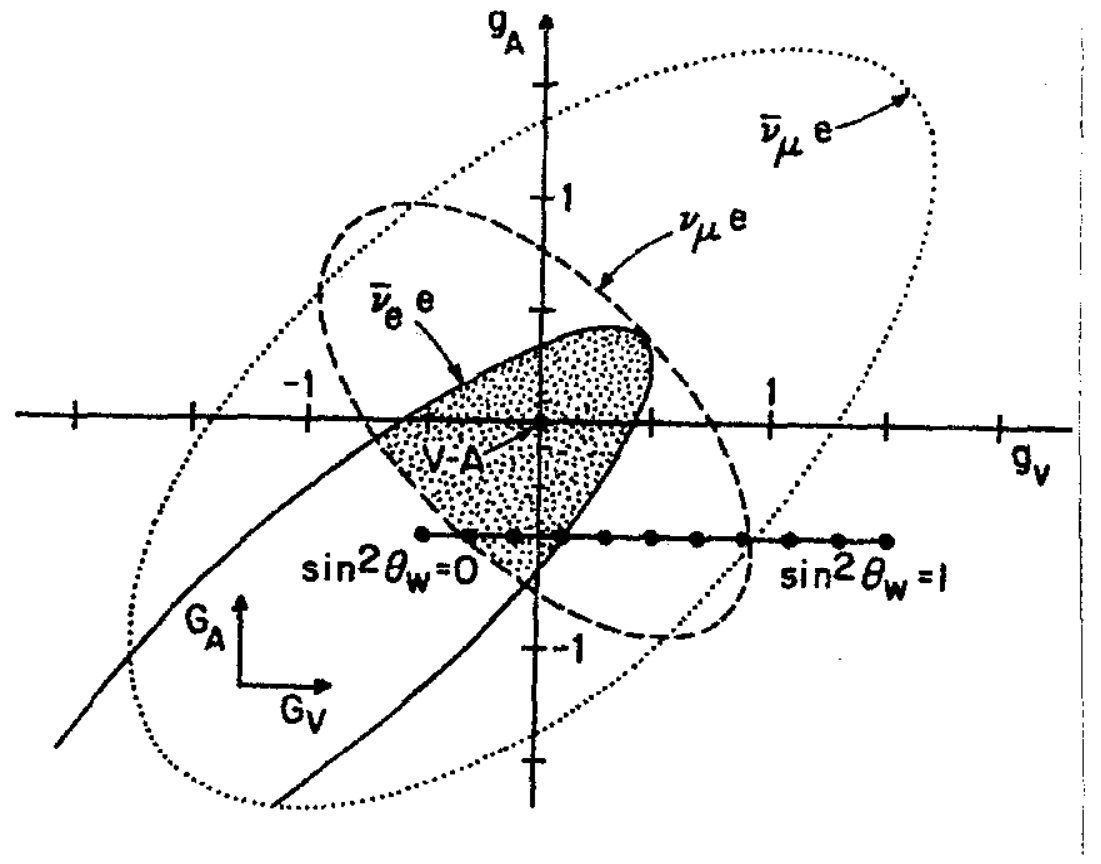

Fig. 15.

Various limits on $\mathrm{g}_{\mathrm{A}}$ and $\mathrm{g}_{\mathrm{V}}$ imposed on by $(v, \bar{v})+\mathrm{e}$ elastic scattering. From A. De Rujula et al., Revs. Mod. Phys, to be published.

5. Neutral Current in Nuclear Physics.

i) Nuclear coherent scattering of neutrinos. Measures the strength of vector current, i. e.,

$$
\begin{gathered}
<\mathrm{I}_{3}-2 \sin ^{2} \theta_{W} \mathrm{Q}>\equiv \text { in } \mathrm{W}-\mathrm{S}_{0} \\
\sigma\left(100 \mathrm{MeV} / \mathrm{c}<q_{\text {recoil }}<300 \mathrm{MeV} / \mathrm{c}\right) \text { on }{ }^{12} \mathrm{C} \\
=\mathrm{a}^{2} \cdot 13.6 \times 10^{-39} \mathrm{~cm}^{2}, \mathrm{E}_{v}>1 \mathrm{GeV} \\
=\mathrm{a}^{2} \cdot 11.2 \times 10^{-39} \mathrm{~cm}^{2}, \mathrm{E}_{v} \approx 200 \mathrm{MeV}
\end{gathered}
$$


[D.Z. Freedman, Phys. Rev. D, to be published.] Also astrophysical implications!

ii) G.T. transitions by $\vec{A}^{3}$. [T.W. Donnelly et al, SLACStanford preprint (1973).]

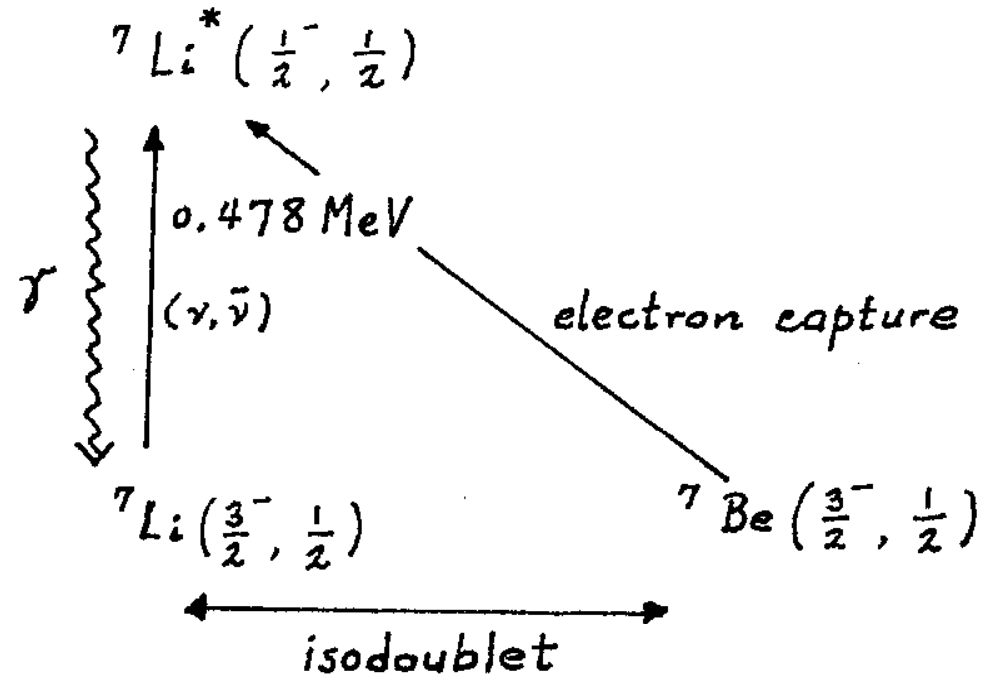

Fig. 16

Schematics of the reaction $(v, \bar{v})+\mathrm{Li} \rightarrow(\nu, \bar{v})+\mathrm{Li}$ $\rightarrow L_{i}+\gamma$ 。 At Savannah River $\left(2 \times 10^{13} \bar{v} / \mathrm{e}^{2} / \mathrm{sec}\right), 4 \gamma^{\prime} \mathrm{s} / \mathrm{day} / \mathrm{Kg}^{7} \mathrm{Li}$ are
expected.

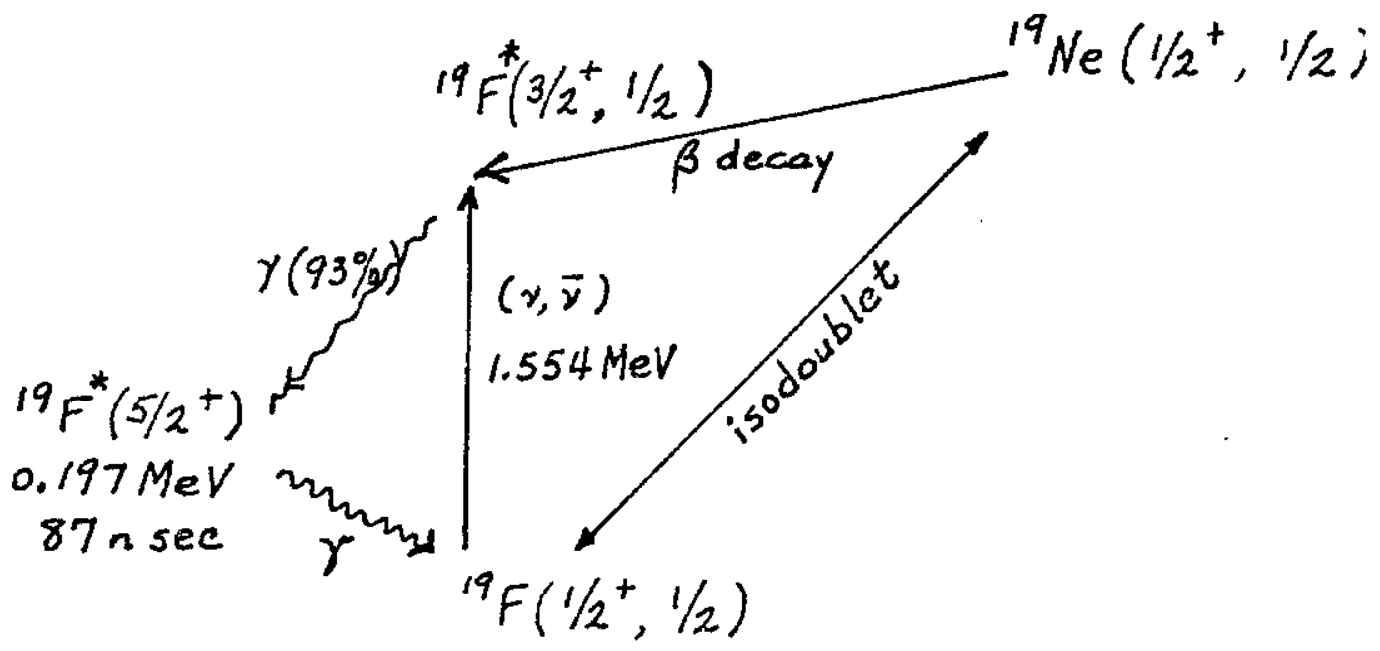

Schematics of $(\nu, \bar{v})+F \rightarrow(v, \bar{v})+F^{*}$ and subsequent decays.

Delayed $2 \gamma$ coincidence gives a unique signature!

iii) Giant dipole excitation. [Bilenky and Dadajan, Dubna 
preprint (1973).] $\left(\mathrm{V}_{0}^{3}\right.$ and $\left.\overrightarrow{\mathrm{A}}^{3}\right)$

$$
\sigma_{v+\mathfrak{v}} \geq\left(1-2 \sin ^{2} \theta_{W}\right)^{2} \sigma_{0} \quad\left(v_{0}^{3} \text { contribution }\right)
$$

where $\sigma_{0}$ can be estimated from photoabsorption cross section. $\sigma_{0}$ on a typical nucleus.
$30 \mathrm{MeV}$
50
100
$10^{-42}$
$10^{-(41 \sim 40)}$
$10^{-39} \mathrm{~cm}^{2}$ 